\title{
Heat Transfer in Segregated Fluidized Beds Part 2: Particle Motion and Its Effects on the Heat transfer in the Segregated Fluidized Beds ${ }^{*}$
}

\author{
${\text { Yihua } \mathrm{GU}^{* *} \text {, Isao SATOH }}^{* *}$, Takushi SAITO ${ }^{* *}$ and Tatsuya KAWAGUCHI ${ }^{* *}$ \\ ${ }^{* *}$ Dept. of Mechanical and Control Engineering, Tokyo Institute of Technology \\ 2-12-1 Oookayama, Meguro-ku, Tokyo, 152-8552 \\ E-mail: gu_yh@mep.titech.ac.jp
}

\begin{abstract}
In our previous paper, particle and temperature segregations in a fluidized bed of binary particle mixtures were experimentally examined, and heat transfer in the segregated fluidized bed was investigated. As the results, it was shown that the temperature segregation results mainly from low heat transfer coefficient through the interface layer, which exists between the flotsam-rich and jetsam-rich layers, and that the heat transfer coefficient increases rapidly with increasing the excess gas velocity.

Following our previous paper, particle motion in the segregated fluidized bed was experimentally investigated in this paper, in order to make quantitative discussion on the relation between the heat transfer coefficient and particle motion in the interface layer. In the experiment, the Particle Imaging Velocimetry (PIV) method was applied to study the concentration and motion of particles in the segregated fluidized bed. A modified solid circulation model was built up to model the particle motion in the segregated fluidized bed.

The experiment results showed that the vertical particle exchange rate of the interface layer increases with the excess gas velocity, and that the vertical heat transfer coefficient through the interface layer is mainly determined by the average particle exchange rate in the interface layer. Variations of the apparent thermal conductivity at different height in the particle layers were also determined by the vertical variation of the particle exchange rate. It was shown that the heat transfer coefficient or the thermal conductivity in the interface layer is influenced by the densities and specific heat capacities of the particles.
\end{abstract}

Key words: Multi-Component Fluidized Bed, Particle Segregation, Temperature Segregation, Particle Motion, PIV, Advection Heat Transfer, Apparent Thermal Conductivity

\section{Introduction}

In the fluidized beds with particle mixture, not only the particle segregation but also the temperature segregation may occur under particular conditions, In order to control the segregation phenomena in practical fluidized beds for heat transfer applications, it is required to know the heat transfer mechanisms dominating the temperature segregation and the effects of particle motion on it. In our previous paper ${ }^{(1)}$, therefore, particle and temperature segregations in a fluidized bed of binary particle mixtures were experimentally examined, and heat transfer in the segregated bed was investigated. As the results, it was shown that the temperature segregation results mainly from low heat transfer coefficient 
through the interface layer, which exists in between the flotsam- and jetsam-rich layers, and that the heat transfer coefficient increases rapidly with increasing the excess gas velocity. In order to make quantitative discussion on the relation between the heat transfer coefficient and particle motion in the interface layer, however, one should know the behavior of particles in the segregated fluidized beds.

In general, heat transfer in fluidized beds under low temperature operation condition consists of conductive heat transfer among particles, convective heat transfer between the particles and gas flow, and heat convection (or advection) due to the particle motion ${ }^{(2)}$. The conduction heat transfer in the stagnant part of the bed is mainly determined by the particle's thermal properties, especially the thermal conductivity, and influenced also by the void fraction of the beds. The convective heat transfer between the particles and gas flow is not very high and kept almost unchanged when the particles are fluidized, for the relative velocity between the solids and gas flow keeps almost constant in the fluidization state. The advection heat transfer is determined by the particle exchange rate which is related to the particle movements. The high particle exchange rate leads to the high advection of heat and the low particle exchange rate leads to the low advection of heat. This means that, in the segregated fluidized beds, coexistence of the stagnant and vigorously fluidized particles at different height leads to the distribution of the conduction and advection heat transfer, and it makes the heat transfer within the segregated fluidized bed much more complicated than that in the mono-component fluidized bed.

There have been a number of literatures related to the particle segregation in fluidized beds of particle mixtures. A conceptual mathematical model for the calculation of segregation patterns was first proposed by Gibilaro and Rowe ${ }^{(3)}$. In their model (G-R model), the bed was assumed to consist of two phases, a wake phase and a bulk phase, and four model parameters, i.e. circulation rate, exchange rate between the phases, segregation rate, and axial dispersion rate in the bulk phase, were defined for the competitive mixing and segregation rate mechanisms. A similar two-parameter (segregation and axial dispersion) model was also proposed by Gel'perin et al. ${ }^{(4)}$. By choosing an appropriate value for each parameter, these models give qualitative agreement with the segregation patterns observed in some typical cases. However, they are not deterministic for predicting segregation patterns for a given mixture, and the relationships between the model parameters and operating conditions are still required. Burgess et al. ${ }^{(5)}$ and Yoshida et al. ${ }^{(6)}$ proposed models to explain unsteady-state segregation behavior, and related the model parameters to the bubbling bed concept ${ }^{(7-16)}$.

However, the mechanisms dominating the temperature segregation in the segregated fluidized beds remains still unclear. The limited applicability of the aforementioned models makes it difficult to predict the particles' behavior in the bed. On the other hand, rapid development of the Particle Imaging Velocimetry (PIV) method and high-speed cameras has made it possible to observe and study the particle motion in fluidized beds directly. This may provide the base for the evaluation of the particle exchange in the segregated bed, which is used to calculate the heat advection in the particle mixture.

In this paper, the particle motion in the segregated fluidized bed, especially in the interface layer between the flotsam and jetsam layers, was experimentally studied by using the PIV method, and its effects on the temperature segregation and heat transfer in the fluidized bed were discussed. A high-speed camera was applied to take successive photographs for the solids, and the photographs were then processed by using the PIV method to get the particle concentration and particle motion. By using the behavior of particle motion in conjunction with the heat transfer characteristics examined in our previous paper, a theoretical model was built up to describe the influences of the superficial gas velocity and the solids' properties on the heat transfer in the segregated fluidized bed. 


\section{Nomenclature}

$A$ cross-sectional area of distributor, $\mathrm{m}^{2}$

$C_{p} \quad$ specific heat at constant pressure, $\mathrm{J} / \mathrm{kg} \cdot \mathrm{K}$

$d_{p}, d_{f}, d_{j} \quad$ particle diameter, flotsam particle diameter, jetsam particle diameter, $\mathrm{m}$

$d T / d Y \quad$ vertical temperature gradient, $\mathrm{K} / \mathrm{m}$

$H \quad$ thickness of the interface layer, $\mathrm{m}$

$h$ heat transfer coefficient, $\mathrm{W} / \mathrm{m}^{2} \cdot \mathrm{K}$

$k$ thermal conductivity, $\mathrm{W} / \mathrm{m} \cdot \mathrm{K}$

$n_{p, S} \quad$ surface number concentration of particles, $1 / \mathrm{m}^{2}$

$n_{p, V} \quad$ volume number concentration of particles, $1 / \mathrm{m}^{3}$

$\mathrm{Nu} \quad$ Nusselt number $\left(=h d_{p} / k_{g}\right)$, dimensionless

$q_{\text {down }}$ downward heat flux, $\mathrm{W} / \mathrm{m}^{2}$

Re Reynolds number $\left(=U d_{p} / k_{g}\right)$, dimensionless

$T$ temperature, $\mathrm{K}$

$U$ superficial velocity or particle velocity, $\mathrm{m} / \mathrm{s}$

$U_{m f}$ minimum fluidization velocity, $\mathrm{m} / \mathrm{s}$
$V \quad$ volume flux, $\mathrm{m} / \mathrm{s}$

$x$ particle fraction, dimensionless

$Y \quad$ vertical coordinate or height, $\mathrm{m}$

\section{subscripts}

down downflow

$f$ flotsam

$j \quad$ jetsam

$g$ gas

$m$ mass

$M \quad$ evaluated from particle motion

$p$ particle

$T$ evaluated from temperature distribution

\section{Greeks}

$\varepsilon \quad$ void fraction, dimensionless

$\rho$ density, $\mathrm{kg} / \mathrm{m}^{3}$

$\Delta T_{H}$ temperature difference in the interface layer, $\mathrm{K}$

$\Delta U$ excess velocity $\left(=U-U_{m f}\right), \mathrm{m} / \mathrm{s}$

$\Delta U_{0}$ maximum excess velocity at which the apparent particle segregation exists, $\mathrm{m} / \mathrm{s}$

\section{Experimental Apparatus and Procedure}

\subsection{Fluidized Bed and Particles}

As is shown in Fig. 1, a fluidized bed was designed to study the characteristics of the particle mixture. The bed size was $0.3 \mathrm{~m} \times 0.1 \mathrm{~m} \times 1.0 \mathrm{~m}$ (Width $\times$ Depth $\times$ Height). Two transparent polymer plates were installed on the fluidized bed as the front and back walls to allow a visible observation. Two perforated aluminum plates gripping a piece of cotton cloth were installed at the bottom of the fluidized bed as a distributor. The gas flow, produced by an air compressor before entering the fluidized bed, was regulated by a valve and measured by a flow meter. The pressure drop on the bed material is measured by a digital manometer to find out the minimum fluidization velocity of the particle mixture. A heater and a cooler were horizontally immersed in the particle layer at the center of the fluidized bed, so as to obtain the corresponding fluidization states to those in our previous paper. The heights of the heater and cooler were $70 \mathrm{~mm}$ and $15 \mathrm{~mm}$ above the distributor, respectively. In the experiment, however, the heater and cooler were not operated.

Several kinds of Geldart B solids were selected to compose the binary mixture. The detailed properties of the particles were shown in Table 1, and the particle mixtures used in the experiment were shown in Table 2. In this paper, the experiment results for the Plastic-Glass06 mixture (mixture A), and the Plastic-Si3N4 mixture (mixture B), were shown. In the segregated fluidized bed with particle mixture B (Plastic-Si3N4), the light Plastic particles have lower $U_{m f}$ and stay at the upper part of the particle mixture, while the Si3N4 particles have higher $U_{m f}$ and stay at the bottom part of the particle mixture. The Plastic and Si3N4 particles are called the flotsam and jetsam, respectively.

\subsection{Measurement Systems}

As is shown in Fig. 2, a high-speed camera, together with a strong illuminant, was used to take successive photos for the particle mixture inside the transparent wall of the fluidized 
bed at the equilibrium state. The selected lens installed on the camera can recognize only the first layer of particles near to the wall but ignore the particles behind the first layer. The particle concentration and particle motion could be calculated out with the PIV method. As the black Si3N4 particles reflect the light and the plastic particle is transparent, the Si3N4 particles can be easily distinguished from the plastic particles. The particle's vertical concentration and movements at different heights were measured by taking photos at different heights.

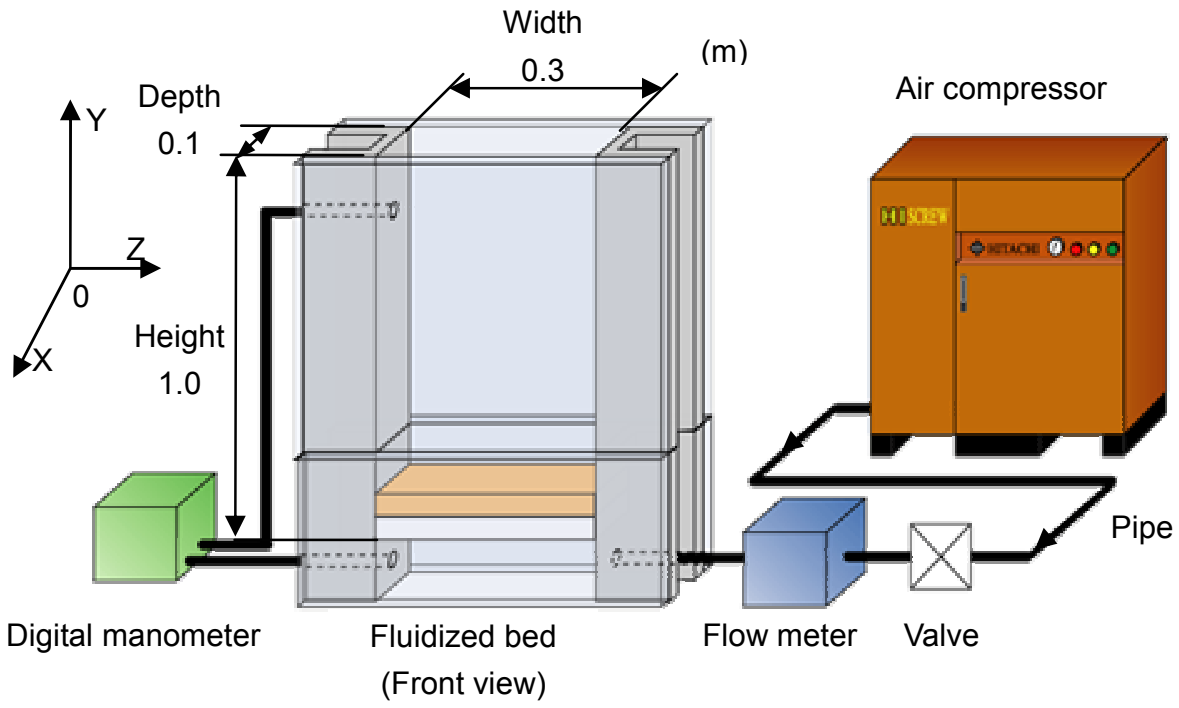

Fig. 1 Fluidized bed system used in the experiment.

Table 1 Particle's properties.

\begin{tabular}{|c|c|c|c|c|c|c|}
\hline Type & $\begin{array}{c}d_{p} \\
(\mathrm{~mm})\end{array}$ & $\begin{array}{c}\rho \\
\left(10^{3} \mathrm{~kg} / \mathrm{m}^{3}\right)\end{array}$ & $\begin{array}{c}C_{\mathrm{p}} \\
(\mathrm{kJ} / \mathrm{kg} \cdot \mathrm{K})\end{array}$ & $\begin{array}{c}k \\
(\mathrm{~W} / \mathrm{m} \cdot \mathrm{K})\end{array}$ & $\begin{array}{c}U_{m f} \\
(\mathrm{~m} / \mathrm{s})\end{array}$ & $\varepsilon$ \\
\hline Plastic & 0.6 & 1.47 & 1.40 & 0.12 & 0.204 & 0.374 \\
\hline Glass06 & 0.6 & 2.47 & 0.75 & 0.65 & 0.241 & 0.397 \\
\hline Si3N4* & 0.5 & 3.22 & 0.71 & 20.00 & 0.278 & 0.361 \\
\hline
\end{tabular}

* Si3N4: $\mathrm{Si}_{3} \mathrm{~N}_{4} 92 \%$

Table 2 Particle mixture.

\begin{tabular}{|c|c|c|c|c|c|}
\hline \multirow[b]{2}{*}{ Particle mixture } & \multicolumn{2}{|c|}{ Flotsam } & \multicolumn{2}{|c|}{ Jetsam } & \multirow{2}{*}{$\begin{array}{c}U_{m f} \\
(\mathrm{~m} / \mathrm{s})\end{array}$} \\
\hline & Type & $\begin{array}{c}\text { Thickness** } \\
\text { (m) }\end{array}$ & Type & $\begin{array}{c}\text { Thickness** } \\
\text { (m) }\end{array}$ & \\
\hline A, (Plastic-Glass06) & Plastic & 0.035 & Glass06 & 0.035 & 0.231 \\
\hline B, (Plastic-Si3N4) & Plastic & 0.040 & Si3N4 & 0.040 & 0.222 \\
\hline
\end{tabular}

**Thickness: the bed height each kind of particle owns in the clearly segregated state

\subsection{Experimental Conditions and Procedure}

In the multi-component fluidized bed, the influence of the original mixing state of particles on the segregation process is remarkable if the superficial velocity is gradually ascended from zero to the prescribed value. However, the influence becomes very small if the superficial velocity is gradually reduced from high superficial velocity to a prescribed value after an enough long time of operation. In this experiment, therefore, the superficial velocity was firstly ascended to $0.45 \mathrm{~m} / \mathrm{s}$ to generate a well-mixed state and then gradually reduced to the prescribed velocity. After an enough long time of operation, typically 15 minutes, at the prescribed superficial velocity, the particle mixture's mechanical and thermal equilibriums were achieved. The measurements were then taken to study the segregation 
behaviors of the particle mixture.

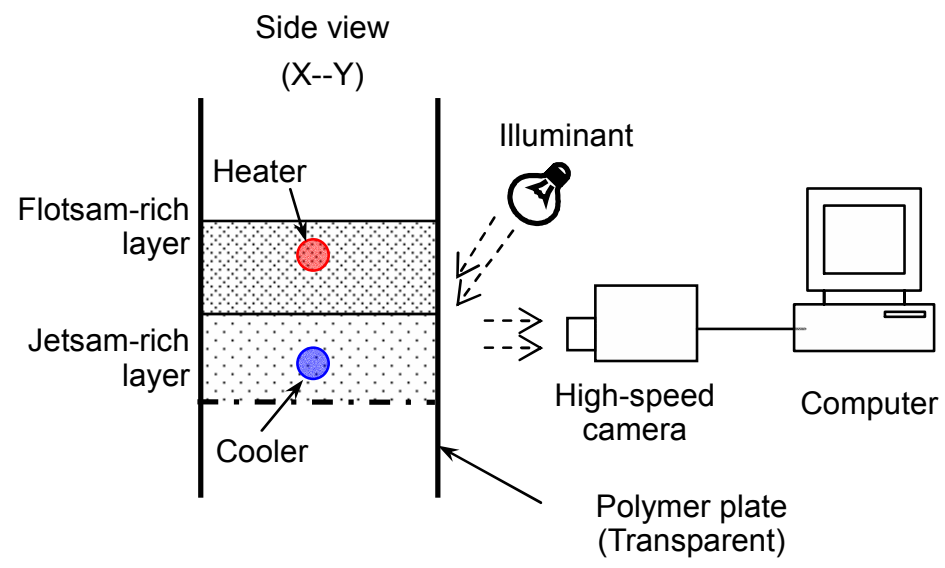

Fig. 2 Apparatus used for particle motion measurement.

\section{Models for Describing the Particle Motion and Heat Transfer}

\subsection{A Model for Particle Circulation}

In the widely used G-R model ${ }^{(3)}$, the bed is assumed to consist of two phases, a wake phase and a bulk phase. Actually, under low and mediate superficial velocities, the bubble's activity in the interface layer of the segregated fluidized bed is not vigorous and particle motion in the interface layer is also limited. Therefore, most of the particles in the interface layer remain static while only part of the particles move upward or downward, as schematically illustrated in Fig. 3.
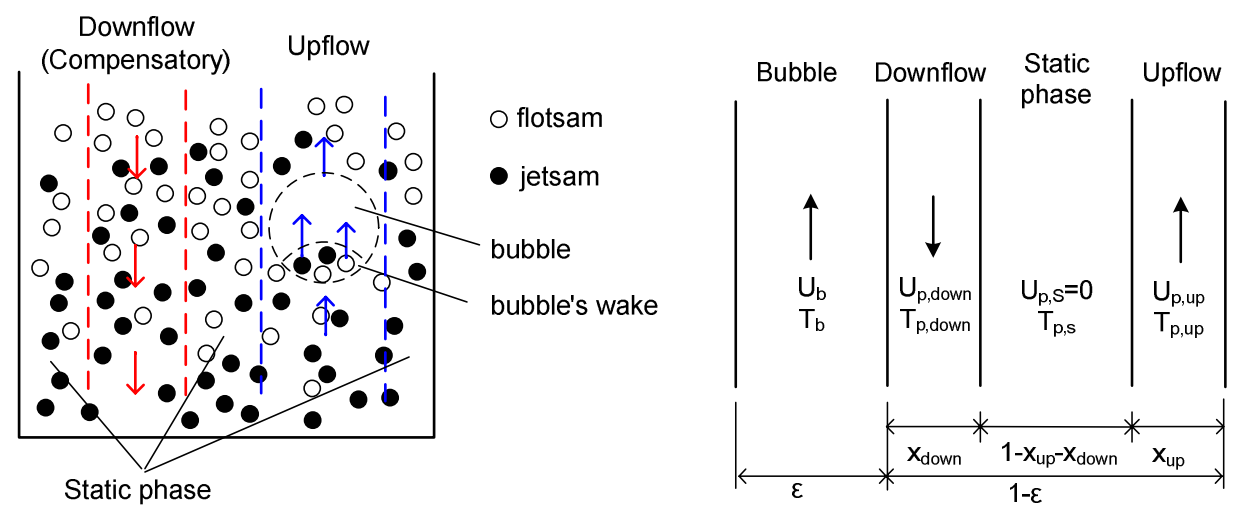

Fig. 3 Particle motion in the multi-component fluidized bed.

Fig. 4 Solid circulation model.

In this paper, a solid circulation model, shown in Fig. 4, is applied to describe the particle motion in the segregated fluidized bed. In this model, the segregated fluidized bed is assumed to consist of three phases: upflow, downflow and static phases. The combination of the upflow and downflow lead to the segregation of the particle mixture and the existence of the interface layer.

\subsection{Volume Fraction of Particles}

The volume fraction $x_{p, V}(p=f, j)$ of particles in the bed can be calculated by using the particle's surface number concentration at the wall side, $n_{p, s}$. For the fixed bed of spherical particles with single diameter $d_{p}$, volume fraction $x_{p, V}$ and surface number concentration $n_{p, S}$ can be derived by assuming that the particles are in the hexagonal close-packed structure as $x_{p, V}=\pi / 3 \sqrt{2}$ and $n_{p, S}=2 / \sqrt{3} d_{p}^{2}$. Thus $x_{p, V}=\left(\pi d_{p}^{2} / 6(\sqrt{2} / \sqrt{3})\right) n_{p, S}$. This is also valid 
for the expanded fluidized particles, if the bed expansion due to fluidization is assumed to exist only in vertical direction, and not to occur in horizontal directions. In addition, the flotsam and jetsam particles used in the experiments have similar size, and thus the particle's volume fraction can be expressed as

$$
x_{p, V}=\frac{\pi d_{p}^{2}}{6 \frac{\sqrt{2}}{\sqrt{3}}} \frac{n_{p, S}}{x_{0}}, p=f, j,
$$

where $x_{0}$ is the particles' recognition factor in the experiment. In this experiment, we assumed $x_{0}$ $\approx 0.9$. By using the Eq. (1), volume fraction of the jetsam/flotsam particles in the segregated fluidized bed can be estimated from the photos captured from a side wall of the bed. According to the definition of the void fraction in the bed, there is a relation that

$$
x_{f, V}+x_{j, V}=1-\varepsilon \text {. }
$$

\subsection{Heat Flow through the Interface Layer}

A model for vertical heat transfer in the segregated fluidized bed is shown in Fig. 5. The heat flux caused by the advection due to particle motion in the segregated fluidized bed is schematically illustrated in Fig. 5(a), and Fig. 5(b) shows the schema of the vertical temperature distribution in the bed, which is based on the temperature distributions measured in our previous study ${ }^{(1)}$. Note that, in this figure, it was supposed that a heater and a cooler are immersed in the flotsam-rich and jetsam-rich layers respectively, corresponding to the experimental apparatus used in our previous paper. In the upflow phase, particles at lower temperature, $T_{L}$, are carried up by the rising bubbles. In the downflow phase, the particles at higher temperature, $T_{H}$, go downward, and the heat transfer takes place from the downflow phase to the upflow phase.
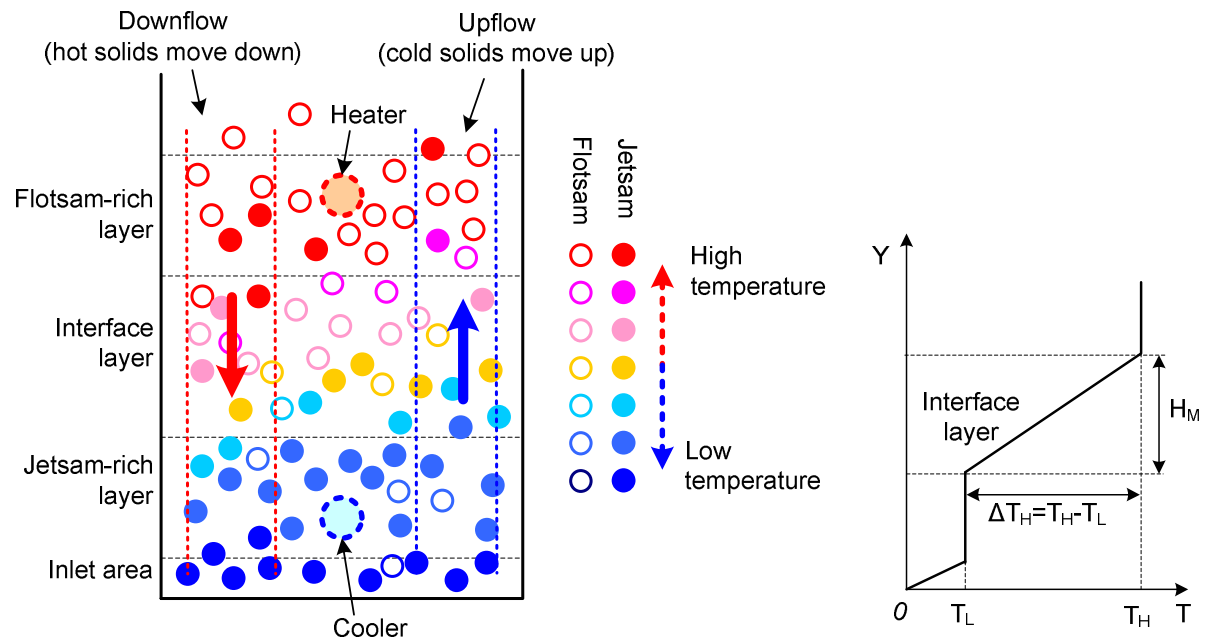

(a) Heat flux caused by particle motion.

(b) Temperature distribution.

Fig. 5 Heat flux in the segregated fluidized bed.

Under the circumstance, the average heat flow through the interface layer, which is located between the flotsam and jetsam layers, caused by the particle motion could be simplified to be the difference between the heat taken down by the downflow and that carried up by the upflow. According to the law of mass conservation in the equilibrium state, the average upward and downward mass flux are equivalent. Thus, the downward heat flux passing through the interface layer due to particle exchange can be estimated as

$$
q_{\text {down }}=\left(\rho_{p} C_{p p}\right)_{\text {aver }} V_{p, \text { aver }} \Delta T_{H},
$$

where $V_{p \text {,aver }}$ the average volume flux of the interface layer in the segregated fluidized bed, and $\Delta T_{H}$ the temperature difference of the interface layer as shown in Fig.5(b). $\left(\rho_{p} C_{p p}\right)_{a v e r}$ is the average heat capacity of the particles per unit volume at the interface layer, and is 
evaluated as $\left(\rho_{p} C_{p p}\right)_{a v e r}=\left(x_{f, V} \rho_{f} C_{p f}+x_{j, V} \rho_{j} C_{p j}\right) /\left(x_{f, V}+x_{j, V}\right)$. Since at the middle of the interface layer, the volume fractions of the jetsam and flotsam particle are almost identical, $\left(\rho_{p} C_{p p}\right)_{\text {aver }}$ can be evaluated simply as

$$
\left(\rho_{p} C_{p p}\right)_{\text {aver }}=0.5\left(\rho_{f} C_{p f}+\rho_{j} C_{p j}\right) \text {. }
$$

\section{Results and Discussions}

\subsection{Particle Motion}

\subsubsection{Fraction of downward particles}

By using the PIV method, the movement of particles in the interface layer was measured. In the mathematical model, we defined the fraction of downward particles as the ratio of the number concentration $n_{p, V, \text { down }}$ of downward particles to the total particle number concentration $n_{p, V}$ at the height.

$$
x_{p, \text { down }}=\frac{n_{p, V, \text { down }}}{n_{p, V}}, p=f, j .
$$

In this study, it was assumed that the fraction of downward particles in the bed was the same as the fraction of downward particles measured at the surface adjacent to the side wall of the bed. That is,

$$
x_{p, \text { down }} \approx x_{p, S, \text { down }}=\frac{n_{p, S, \text { down }}}{n_{p, S}}, p=f, j,
$$

where $n_{p, S \text {, down }}$ is the average surface number concentration of downward particles at the wall side. The fraction of upward particles was evaluated in the same manner.

Figure 6 shows the fractions of the upward and downward jetsam particles in the mixture B (Plastic-Si3N4) evaluated from the particle motion captured in the experiment. In the segregated fluidized bed, most of the particles in the fluidized bed remain static or just "shake," and thus the fractions of the upward or downward jetsam particles are small. As is shown in Fig. 6, the fractions of both the upward and downward jetsam particles increase with the excess gas velocity $\Delta U\left(=U-U_{m f}\right)$ at low excess velocities, and also depend on the height in bed. In the interface layer (typically $Y=0.03 \sim 0.05 \mathrm{~m}$ ), the fraction of downward jetsam particles $x_{j \text {,down }}$ is almost proportional to $\Delta U$, and the average fraction of the downward jetsam particles in the interface layer is near to $x_{j, \text { down }}$ in the middle height of the interface layer $(Y \approx 0.042 \mathrm{~m})$ as shown in Fig. 6 . Under the experimental conditions, the relation between the average fraction $x_{j, \text { down }}$ and the excess velocity $\Delta U$ for the interface layer can be empirically correlated as

$$
\frac{x_{j, \text { down }}}{\Delta U} \approx 1.5\left(m^{-1} s\right) \text {. }
$$

\subsubsection{Average velocity of downward particles}

The average velocity of downward jetsam particles in the mixture B was shown in Fig. 7. Generally, at each height, the average velocity of the jetsam particles increases with $\Delta U$, and the jetsam particle at higher locations has higher downward velocities. One can find from Fig. 7 that the average velocity of downward jetsam particles can be correlated with the excess velocity $\Delta U$ by representatively using the data at the middle height of the interface layer $(Y \approx 0.042 \mathrm{~m})$ as

$$
\frac{U_{j, \text { down }}}{\Delta U} \approx 1 \text {. }
$$

In the interface layer, although the jetsam particle has the priority of falling than the flotsam particles when bubbles passed by, it was observed that the most of the flotsam particles have the similar motion with the nearby jetsam particles. Therefore, the downward flotsam particles are assumed to have the same downward velocity with the downward jetsam particles at the same height. The fraction of downward flotsam particles is also 
assumed to be same as the fraction of jetsam particles at the same height. Thus,

$U_{f, \text { down }} \approx U_{j, \text { down }}$
$x_{f, \text { down }} \approx x_{j, \text { down }}$.

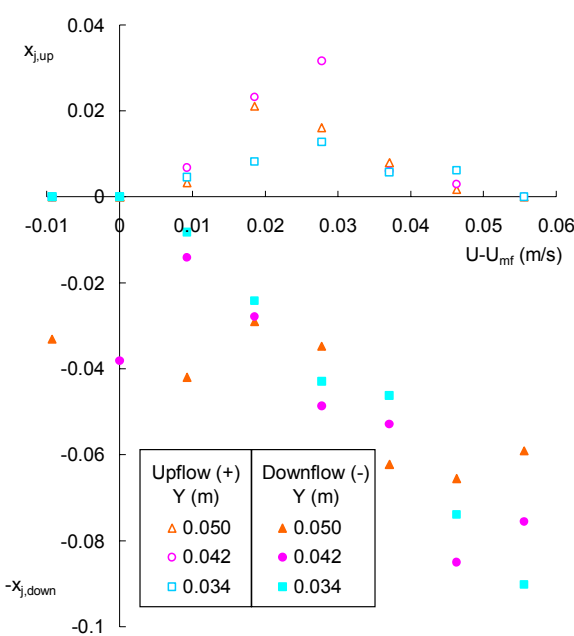

Fig. 6 Fraction of the upward and downward jetsam particles (plastic-Si3N4).

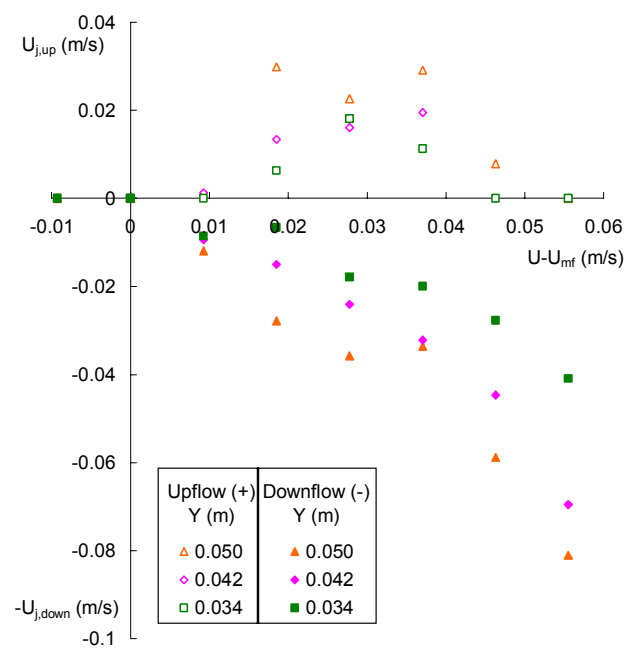

Fig. 7 Average velocity of the upward and downward jetsam particles (plastic-Si3N4).

\subsubsection{Vertical volume exchange rate of the particles}

With the particle motion modeled above, the downward volume flux of particles is then expressed as

$$
V_{p, \text { down }}=x_{p, V} x_{p, \text { down }} U_{p, \text { down }}, p=f, j .
$$

By combining the Eqs. (2), (9), (10) and (11), the total vertical volume flux, or the vertical volume exchange rate, of the binary mixture can be estimated as the sum of the downward volume fluxes of the jetsam and flotsam particles. That is,

$$
V_{p, \text { aver }}=V_{f, \text { down }}+V_{j, \text { down }} \approx(1-\varepsilon) x_{j, \text { down }} U_{j, \text { down }} \text {. }
$$

Figure 8 shows the volume exchange rate of particles in the segregated fluidized bed of the mixture B evaluated from the experimental results. As shown in this figure, the vertical particle exchange rate varies with the excess velocity $\Delta U\left(=U-U_{m f}\right)$ as follows:

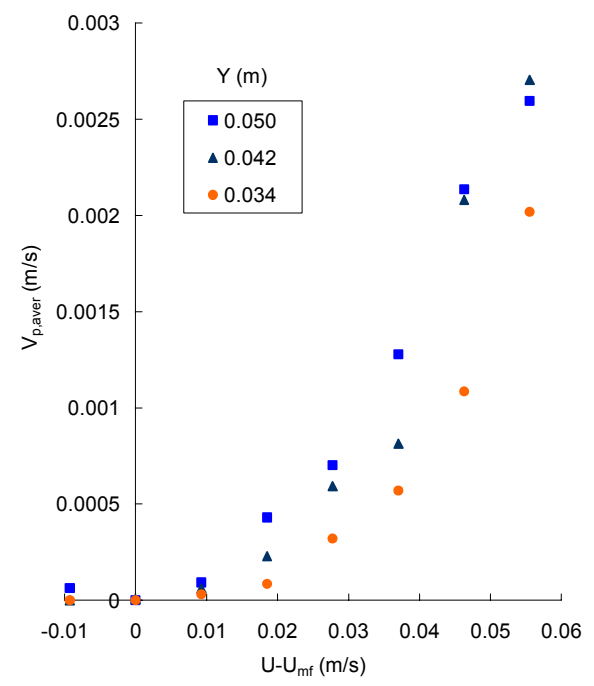

Fig. 8 Volume exchange rate of particles in the interface layer (plastic-Si3N4).

(1) $\Delta U \leq 0$ : The jetsam-rich layer remains static. Little particle exchange takes place between the jetsam-rich and flotsam-rich layers. The movements of the jetsam particles in the interface layer are limited, and only few of them come up into the flotsam-rich layer.

(2) $0<\Delta U<\Delta U_{0}$ : The jetsam-rich layer is partially fluidized, and the movements of the 
jetsam particles and the particle exchange rate in the interface layer are improved. Under the experimental conditions, the $\Delta U_{0}$ of the particle mixture B was typically $0.04 \mathrm{~m} / \mathrm{s}$. In our previous paper, it was shown that the thickness of the interface layer, which is the main region where the jetsam and flotsam particle exchange happened, also increases with the excess gas velocity in this range. As shown in this figure, however, the particle exchange rate is limited in this velocity range. This suggests that the heat transfer due to solid convection would be also limited in the interface layer.

(3) $\Delta U \geq \Delta U_{0}$ : The interface layer has extended to the full height of the bed except for the inlet area. Under the situation, the particle exchange in the mixture becomes very high.

\subsection{Relation between Particle Exchange Rate and Heat Transfer in the Interface Layer}

\subsubsection{Average vertical heat transfer coefficient of the interface layer}

Using the characteristics of the particle motion mentioned above, one could estimate the average vertical heat transfer due to particle advection in the interface layer. The average heat transfer coefficient $h_{M}$ is evaluated from the motion of the downward particles in the middle of the interface layer $(Y \approx 0.042 \mathrm{~m})$ as follows.

$$
h_{M}=\frac{q_{\text {down }}}{\Delta T_{H}}=(1-\varepsilon)\left(\rho_{p} C_{p p}\right)_{\text {aver }} x_{j, \text { down }} U_{j, \text { down }}
$$

The average vertical heat transfer coefficients due to particle advection and the Nusselt numbers evaluated by using the particle motion measured in this study are shown in Figs. 9 and 10. The Nusselt number is defined as

$$
N u_{M}=\frac{h_{M} d_{p, \text { aver }}}{k_{g}}
$$

where $d_{p, a v e r}$ shows the average diameter of particles, and is $d_{p, a v e r}=1 /\left(x_{j, m} / d_{p, j}+x_{f, m} / d_{p, f}\right)$.

In these figures, the heat transfer coefficient $h_{\mathrm{T}}$ and $N u_{\mathrm{T}}$ estimated from the temperature measurement described in our previous paper are also shown for the reference purpose. One can see in Fig. 9 that the heat transfer coefficient $h_{M}$ estimated from the particle motion agrees very well with that evaluated from the temperature distribution in the segregated fluidized bed. As the $\varepsilon$ and $\left(\rho_{p} C_{p p}\right)_{a v e r}$ are almost constant in the particle mixture at low excess velocities, and $x_{p, \text { down }}$ and $U_{p, \text { down }}$ of the interface layer are proportional to $\Delta U$ as shown in the previous section, $h_{M}$ is proportional to the square of the excess velocity $(\Delta U)^{2}$. This corresponds the fact that the heat transfer coefficient in the interface layer of the segregated fluidized bed is proportional to the square of the excess velocity, which has been revealed in our previous paper.

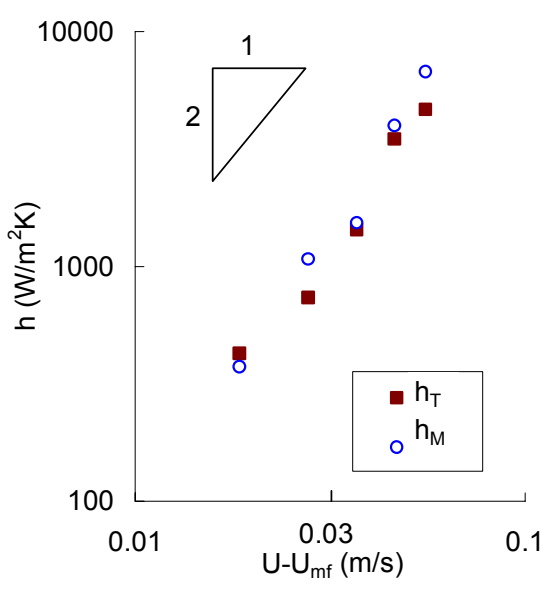

Fig. 9 Average vertical heat transfer coefficient of the interface layer (plastic-Si3N4).

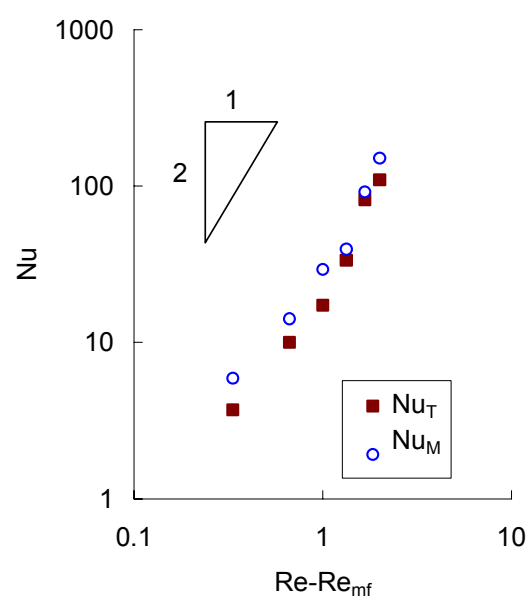

Fig. 10 Nusselt number of the average heat transfer of the interface layer (Plastic-Si3N4). 
Equation (13) means that the heat transfer coefficient of the interface layer is determined by the particle exchange rate of the interface layer and the particles' densities and specific heat capacities. In this experiment, the particle mixture A (Plastic-Glass06) has the similar $\left(\rho_{p} C_{p p}\right)_{\text {aver }}$ with the mixture B (Plastic-Si3N4), i.e. $\left(\rho_{p} C_{p p}\right)_{\text {aver, } A} /\left(\rho_{p} C_{p p}\right)_{\text {aver }, B} \approx 0.9$. Therefore, the heat transfer coefficients through the interface layer in these mixtures are similar when the particle exchange is similar, as is proved in previous paper.

Using the heat transfer coefficient through the interface layer and the thickness of the interface layer, the apparent thermal conductivity $k_{M}$ of this layer can be estimated as follows:

$$
k_{M}=h_{M} H_{M},
$$

where $H_{M}$ is the thickness of the interface layer. In Fig. 11, the apparent thermal conductivity estimated from the particle motion is compared with that calculated by using the measured temperature distribution ${ }^{(1)}$. One can find from this figure that the apparent thermal conductivity of the interface layer estimated from the particle motion agrees very well with that evaluated by using the temperature distribution in the bed, and that the thermal conductivity is almost in proportion to the cubic of the excess velocity $(\Delta U)^{3}$. This is because that, not only the particles' exchange rate, the region where the particle exchange occurs expands almost linearly as the excess velocity increases.

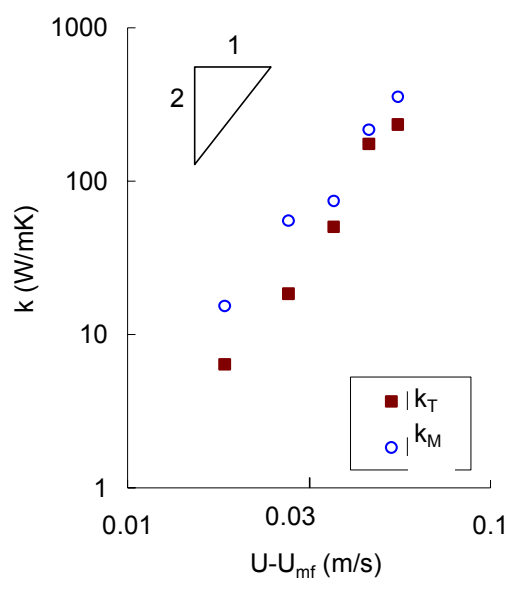

Fig. 11 Apparent thermal conductivity of the interface layer (Plastic-Si3N4).

\subsubsection{Vertical distribution of the thermal conductivity}

Vertical distributions of the fraction and the average velocity of the downward particles in the whole bed height are shown in Figs. 12 and 13, and the vertical distribution of the vertical volume exchange rate of the particles, which is the product of the fraction and the average velocity, is shown in Fig.14. The data show great scatter due to particle-by-particle measurement of the particle motion, but one can see the perspective tendency that the vertical volume exchange rate of the particle mixture differs with height, and the exchange rate in the jetsam-rich layer is much lower than those in the flotsam-rich and interface layers. In the interface layer, i.e. typically $Y=0.03 \sim 0.05 \mathrm{~m}$, the volume exchange rate decreases as height decreases. It means that only a few hot flotsam particles in the heated flotsam-rich layer go to the cold jetsam-rich layer through the interface layer, and thus little heat exchange takes place between the jetsam-rich and flotsam-rich layers due to particle motion. Therefore, it can be concluded that the apparent vertical thermal conductivity of the interface layer is greatly weakened by the particle segregation.

It should be noted that, as shown in Fig. 14, the exchange rate of the particle has a distinct "dip" on the top of the interface layer, at which the height is about $0.055 \mathrm{~m}$, especially at low excess velocity condition. This tendency results not from the particle velocity (Fig.13), but from the volume fraction of the particle (Fig.12). Under the low excess velocity condition, particles in the jetsam layer still remains almost to be fixed, even 
while the particles in the flotsam layer are fluidized vigorously. Under the situation, gas bubbles tends to generate mainly on the top of the interface layer, and this results in the reduction of particle fraction, or increase in void fraction, in this location as shown in Fig. 12. Reduction of particle fraction at the top of interface layer reduces the heat transfer coefficient through the interface layer. This explains the experimental result of our previous paper that, under the low excess velocity condition, the apparent thermal conductivity of the interface layer is lower than that of the jetsam-rich layer, even though the jetsam layer in the segregated fluidized bed remains almost stagnant.

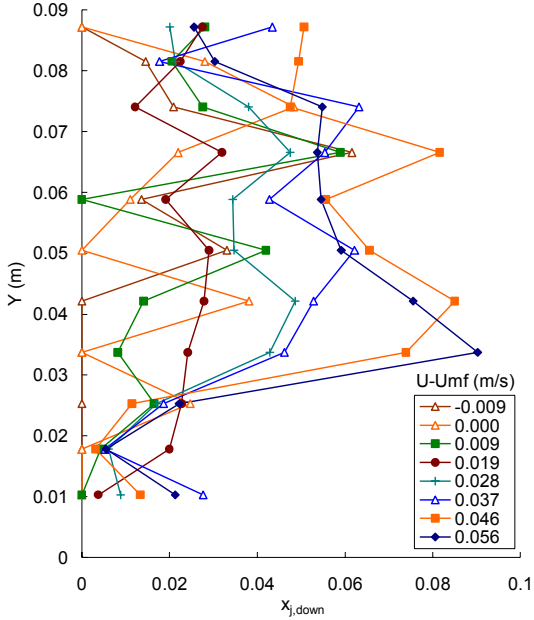

Fig. 12 Fraction of downward particles. (plastic-Si3N4)

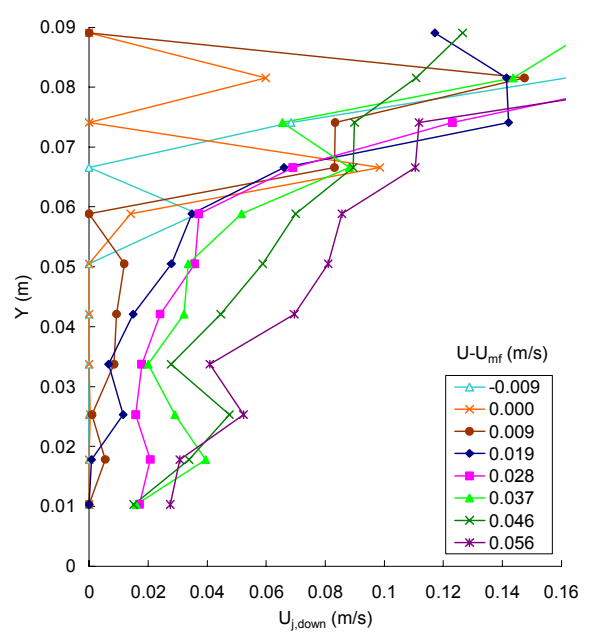

Fig. 13 Vertical velocity of the downward particles. (plastic-Si3N4)

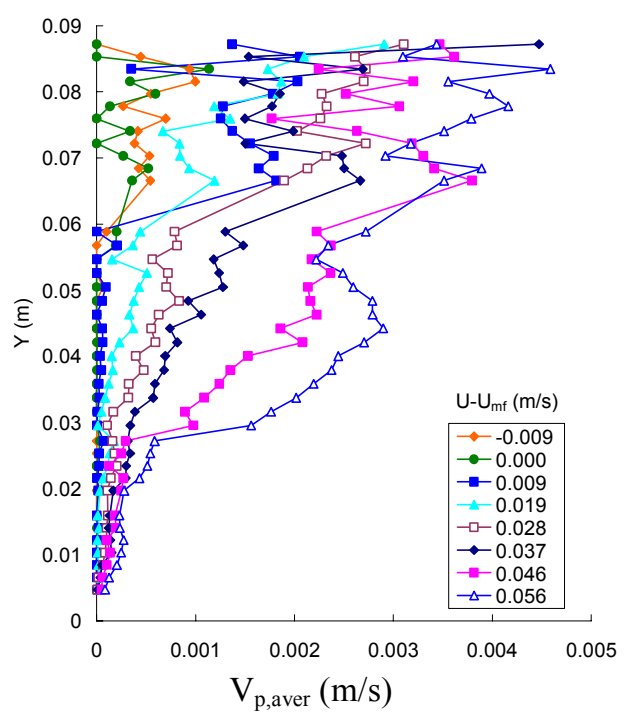

Fig. 14 Vertical distribution of the volume exchange rate (plastic-Si3N4).

\section{Conclusions}

In this paper, particle motion in the segregated fluidized bed was examined by using the PIV method in order to discuss the relation between the particle behavior and the heat transfer characteristics in it. The modified counterflow solid circulation model was built up to study the influence of the particle motion on the heat transfer especially in the interface layer, and it was shown that the model in combination with the measured particle motion behavior explains well the heat transfer characteristics of the interface layer.

In the segregated fluidized bed, it was shown that both the average velocity and the 
fraction of downward particles increase almost linearly with the excess gas velocity, and thus the vertical volume exchange rate of the particles at the interface layer increases with the square of the excess gas velocity. The heat transfer coefficient through the interface layer, which is dependent upon the volume exchange rate of the particles, also increases with the square of the excess gas velocity, and therefore, the thermal resistance of the interface layer rapidly becomes small as the excess velocity increases, and the temperature segregation in the bed disappears consequently.

Under the low excess velocity condition, the volume fraction of downward particles is smaller at the top of the interface layer than in other region, and this results in the reduction of heat transfer through the interface layer. This is the reason why the apparent thermal conductivity of the interface layer is lower than that of the jetsam-rich layer under the low excess velocity condition, even though the jetsam layer in the segregated fluidized bed remains almost stagnant.

\section{References}

(1) Y. Gu, I. Satoh, T. Saito, T. Kawaguchi, Heat transfer in segregated fluidized beds, Part 1, J. Therm. Sci. Tech., 2 (1) (2007) 43-54.

(2) J.F. Davidson and D. Harrison (Eds.), Fluidization, Academic Press, London (1971), 303-306.

(3) L.G. Gibilaro, P.N. Rowe, A model for a segregating gas fluidized bed, Chem. Eng. Sci., 29 (1974) 1403-1412.

(4) N.I. Gelperin, V.V. Zaklarenko, V.G. Ainstein, Teoreticheskie Osnovy Khim. Tech., 11 (1977) p. 572.

(5) J.M. Burgess, A.G. Fane, C.J.D. Fell, Proc. 2nd Pacific Chem. Eng Cong. Denver, U.S.A., Vol. I (1977) p.1405.

(6) K. Yoshida, H. Kameyama, F. Shimizu, Proc. 3rd Eng. Found. Conf. Fluidization, Henniker, U.S.A., (1980) p.389.

(7) L. Chenug, A.W. Nienow, P.N. Rowe, Minimum fluidization velocity of a binary mixtutre of different sized particles, Chem. Eng. Sci., 29 (1974) 1301-1303.

(8) S. Chiba, T. Chiba, A.W. Nienow, H. Kobayashi, The minimum fluidization velocity, bed expansion and pressure drop profile of binary particle mixtures, Powder Technol., 22 (1979) 255-269.

(9) K. Noda, S. Uchida, T. Makinno, H. Kamo, Minimum fluidization velocity of binary mixture of particles with large size ratio, Powder Technol., 46 (1986) 149-154.

(10) A.W. Nienow, N.S. Naimer, T. Chiba, Studies of segregation/mixing in fluidized beds of different size particles, Chem. Eng. Sci., 62 (1987) 52-63.

(11) P.N. Rowe, A.W. Nienow, A.J. Agbim, The mechanism by which particle segregate in gas fluidized beds-binary systems of near spherical particles, Trans. Inst. Chem. Eng., 50 (1989) 310-323.

(12) E. Ekinci, H. Atakul, M. Tolay, Detection of segregation tendencies in a fluidized bed using temperature profiles, Powder Technol., 61(1990) 185-192.

(13) A.C. Hoffmann, L.P.B.M. Janssen, J. Prins, Particle segregation in fluidized binary mixtures, Chem. Eng. Sci., 48 (1993) 1583-1594.

(14) G.R. Mohammad, R. Victor, C. Milan, Segregation potential in binary gas fluidized beds, Powder Technol., 103 (1999) 175-181.

(15) R.C. Wang, C.C. Chou, Particle mixing/segregation in gas - solid fluidized bed of ternary mixtures, Can. J. Chem Eng., 73 (1995) 793-799.

(16) S.Y. Wu, J. Baeyens, Segregation by size difference in gas fluidized beds, Powder Technol., 98 (1998) 139-150. 